\title{
Retinal Disease Detection Using Machine Learning Techniques
}

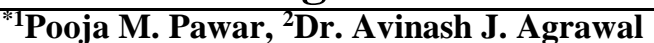

${ }^{1,2}$ Shri Ramdeobaba College of Engineering and Management, Nagpur, Maharashtra, India Email: "pooja27pawar@gmail.com,agrawalaj@rknec.edu

\section{Received: 09 ${ }^{\text {th }}$ July 2018, Accepted: $14^{\text {th }}$ August 2018, Published: $3^{\text {st }}$ August 2018}

\begin{abstract}
One of the complications of diabetes is Diabetic Retinopathy. It occurs when high blood glucose damages the small retinal vessels which provide nutrients and oxygen to the retina. Initially, the diabetic retinopathy treatment results were recorded on the basis of Fundoscopic Exam. It is essential to have an automatic detection method for DR detection at an early stage to prevent severe vision loss. This paper provides a method for DR detection from retinal fundus image using the advanced machine learning techniques. Here we developed the screening system by performing classification using three different classifiers. The performance of proposed work has been tested by using MESSIDOR database. The input images are classified into three classes named Normal, NPDR and PDR. The original dataset has 1047 image, out of which 210 samples were used for testing. The proposed method achieves an accuracy of $74.28 \%$ using Decision tree classifier for multiclass classification and $82.85 \%$ on binary classification.
\end{abstract}

Keywords: ANN, Classification, Decision Tree, Diabetic Retinopathy, Fundus, Machine Learning.

\section{Introduction}

Diabetic retinopathy (DR) is a rigorous and global eye disease which results in blindness and severe vision loss among the working age population. According to World Health Organization (WHO), approximately $10 \%$ population has diabetes and it will rise to 300 million around the world by 2025 . In order to decrease this burden, it requires regular screening and early diagnosis.

There are some distinct features in DR such as abnormality and leakiness of retinal blood vessels. When it moderately grows different lesions appear as microaneurysm, hard exudates, hemorrhages, cotton wool spots and red lesions etc. There are some tiny red dots around retinal blood vessels known as microaneurysms and the bigger blood clots are called as hemorrhages. Exudates are the important components which give information about diabetic retinopathy in primary phases. Exudates are again categorized into two classes named hard exudates and soft exudates (often called cotton wool spots). Ophthalmologists categorize DR into two major classes as Non-proliferative DR (NPDR) and proliferative DR (i.e. PDR). Microaneurysms and hemorrhages called red lesions belong to NPDR.
Neovascularization occurs in PDR where the new abnormal blood vessels generated in the retina.

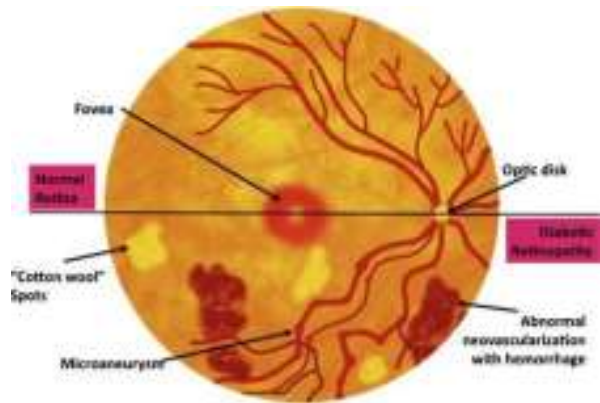

Figure 1. Retinal Image with Features.

Diabetic retinopathy can be identified by orderly screening of retina for examination of its distinct features. But this manual process is time-consuming and is not optimal for large population areas. Hence modern procedures confront the problem by using computer-aided diagnosis (CAD). The motive of this work is to present and develop an automated framework for recognition and classification of diabetic retinopathy stages into PDR, NPDR and Normal of any retinal image. Initially, the input retinal fundus image is pre-processed by grayscale conversion and contrast enhancement, later the features are extracted and the feature vector is generated. Finally, the machine learning models have been applied for classification of input images. The following diagram Figure 1 depicts all the pathological signs of diabetic retinopathy.

\section{Related Work}

Recent developments include the image processing techniques for detection of DR abnormalities and classification. G. G. Gardner et al. [1] used median filtering method for lesion detection and artificial neural network for classification for binary classification of retinal images. D. Vallabha et al. [2] developed a system for automated identification and classification of input retinal vessels by making the use of scale and orientation selective Gabor filter banks. Sohini Roychowdhury et al. [3] detected blood vessels in retinal images and used matched filter for signal detection for detecting piecewise linear segments of retinal blood vessels. They created 12 distinct templates for vessel segments search along with all possible directions. Two-step classification concept was used for red and bright lesion detection. 
Diego Marine et al. [4] have used vessel central light reflex removal, background homogenization and vessel enhancement as a preprocessing method before extraction of vessels from fundus image. Sopharak et al. [5] have developed an automated DR detection technique by using a set of different adjusted morphological operators and thresholding operation. R. Geetha Ramani et al. [6] suggested an approach for extracting the shape feature like perimeter, area, orientation but without performing the segmentation. Resultant shape feature is then evaluated by using various classifiers such as AdaBoostM1. Gwenole Quellec et al. [7] presented an optimal filter for target lesion detection in the input image. Reference optimal filter is used for classifying a new image, it distinguished the lesion in the image. Noise reduction was done for improving the performance and normalization implemented for increasing the sample. Jaydeep De et al. [8] developed the recent graph theory method for the detection of various filamentary deformities in the input image. The thread structure is then separated into disjoint of the thread, the work also includes the segmentation process. The segmented image is converted into skeleton map and digraph is constructed. Finally, for the label propagation purpose, the theorem named matrix-forest was used for tracing on the generated digraph.

All the previous mentioned implemented work included image processing techniques like thresholding and filter processing for classification of the input eye images. These processes achieved great significance but for critical images having many features requires more processing and it may affect the accuracy because of the use of various morphological operators. The following section includes the proposed framework in which we have developed an advanced machine learning concept for the detection of retinal eye disease.

\section{Proposed Method}

This paper provides machine learning techniques for disease classification based on the features of the input image. Figure 2 shows the flowchart of the proposed work. The retinal images used for testing and evaluation were obtained from the publically available DR database named as MESSIDOR. The images are stored in TIFF format having 45-degree Field of View (FoV). First, the input image is given to the pre-processing for normalization and noise reduction. In the second step, segmentation was implemented on the pre-processed images to get various lesions from the input image and GLCM was used for feature vector generation. Finally, in the third step, machine learning classifiers such as Decision Tree, AdaBoost, and Logistic Regression and Neural Network are applied to get the classification results. The whole work in this paper is implemented using PYTHON 3.6 using open source distribution framework called Anaconda. Image processing operations are performed using OpenCV package.
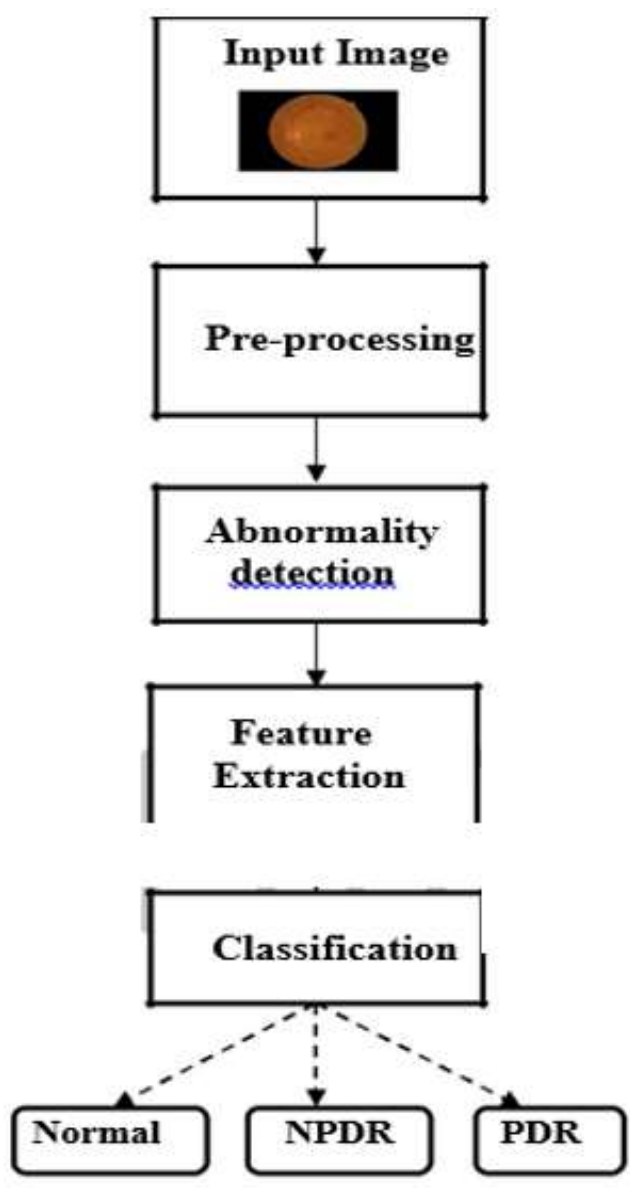

Figure 2. Proposed Method Flowchart

\section{Pre-processing}

Primarily, the input retinal images are pre-processed to remove the irregularities and lighting inconsistencies. The main aim of this step is to eliminate noise from the image. The input image is in RGB (Red, Green, Blue) format as it is a color image given to the system.

\subsection{Grayscale Conversion}

The obtained images are in TIFF format. The color image of RGB format is converted to grayscale. Grayscale conversion is necessary for identifying important edges or other features.

\subsection{Contrast enhancement}

Contrast enhancement is nothing but changing the image value distribution to cover the wider range. Input images obtained by fundus cameras are of varying contrast and having non-uniform lighting, therefore, it is required to enhance the contrast level for lesion detection. Histogram equalization is used for enhancement and here Contrast Limited Adaptive Histogram Equalisation (CLAHE), was applied. CLAHE performs the operation on smaller parts of the image known as tiles. It computes multiple 
histograms, each matches to a single part of the image and applies to realign the intensity values of the image. The result of CLAHE is shown in Figure 3.
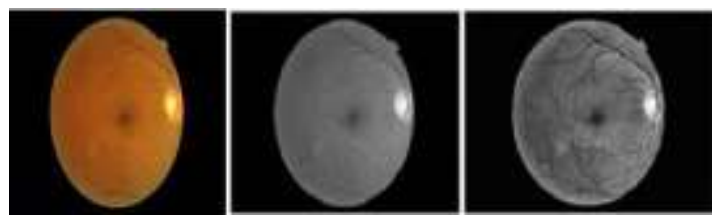

Figure 3. Conversion of RGB Image into Gray Scale and CLAHE

\section{Abnormality detection}

In Diabetic retinopathy, retinal images have various features such as blood vessels, exudates, microaneurysms, and hemorrhages. These features need to be segmented from the input image for feature vector creation. In this paper, blood vessels, microaneurysms, and optic disc have been segmented by using morphological operations.

\subsection{Blood vessels extraction}

Retinal blood vessels are essential features which help evaluation. These vessels exhibit various symptoms when an eye has a certain disease. In diabetic retinopathy, there are swellings in the blood vessels. In case of non-proliferative DR, narrow blood vessels do not get enough blood supply because of blockages occur near optic nerve and it is the reason they release fluid in the retina. On the contrary, new blood vessels are developed in the retina in proliferative DR. Hence its detection is very important because blood vessels are the primary lesions. Image processing operations along with alternate sequential filtering have been used. The input image is split into its RGB format and green channel of an image has been extracted because of its greater contrast. Another reason for green channel extraction is that the presence of blood clots and hemorrhages made it difficult for segmentation. Contrast Limited Adaptive Histogram Equalization was used for contrast improvement. Later, morphological closing and opening operations applied in an alternative sequence, with an ellipse as structuring element. Then the resultant image was subtracted from CLAHE image. Thresholding was applied to binarize the image with a suitable threshold value. The binary image could have some noise and to remove this, morphological erosion was applied. Finally, unwanted pixels were removed to get the desired vessel results. Blood vessel extraction steps are shown in Figure 4.
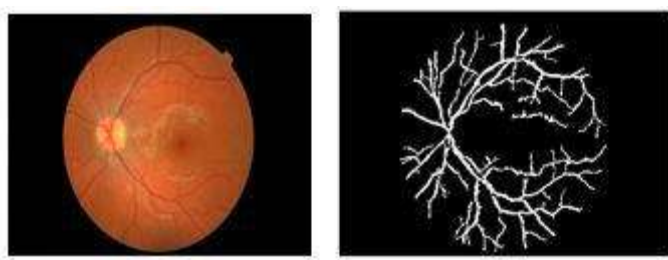

Figure 4. Blood Vessel Detection

\subsection{Microaneurysm detection}

Microaneurysms detection plays an important role in the automatic diagnosis of diabetic retinopathy. These microaneurysms appear as small unique patterns in a circular shape around the blood vessels. For discovering the number of MA, first the green component is extracted from the RGB input image, then this image is converted to grayscale. The morphological opening operation is performed on this image with the disk as structuring element. Later the image is inverted and the opening is applied to get the final microaneurysm image as shown in Figure 5.
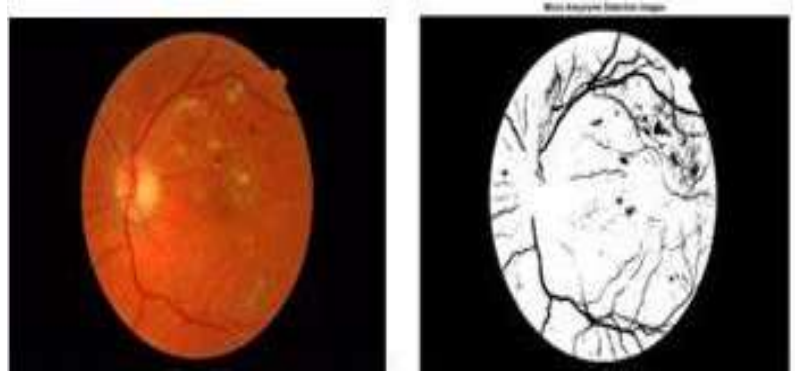

Figure 5. Microaneurysm Detection

\subsection{Exudates detection}

Detection of hard exudates is an essential task for classification. The optic disc is one of the brightest components in the human retina. Green channel of the input image is taken and histogram equalization was applied to this channel. The image was then inverted and thresholding was applied. The presented procedure is shown in Figure 6.
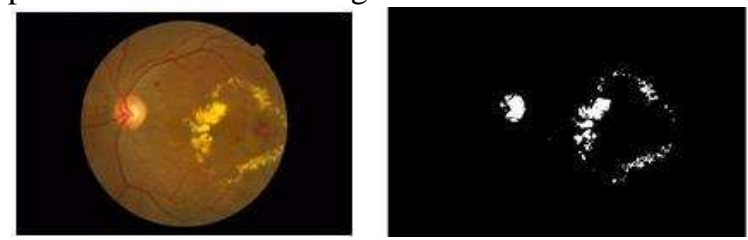

Figure 6. Exudates Detection

\section{Feature Extraction}

For classification of an image into its different classes, it is necessary to represent the image in the form of the feature vector. Feature extraction is nothing but getting the unique features of segmented images. In order to get the important features from the segmented images, a two-dimensional matrix named as a Grey-Level Co-occurrence Matrix (GLCM) is used. It is a statistical method of observing the texture of an image which looks for the spatial relationship of image pixels. GLCM is also named as the gray-level spatial dependence matrix. Here, we have applied "greycomatrix" and "greycoprops" commands in PYTHON to get the following features from the segmented images. Contrast: It gives a measure of the intensity value of contrast between a pixel and its neighborhood pixels in the image. It is represented as: 
I. Contrast: It gives a measure of the intensity value of contrast between a pixel and its neighborhood pixels in the image. It is given by:

Contrast $=\Sigma P_{i},(i-j)_{2 N-1 i, j=0}$

II. Energy: Energy feature corresponds to the sum of squared elements of the image. It is also called the angular second moment or uniformity.

Energy $=\Sigma(P i, j) 2 N-1 i, j=0$

III. Homogeneity: This feature is used to estimate the closeness of the distribution of the components in GLCM matrix to diagonal.

Homogeneity $=\Sigma[P(i, j)][1+|i-j| 2] N-1 i, j=0$

IV. Correlation: The correlation operation is similar to convolution operation. Here, the output value of a pixel is calculated as a sum of neighboring pixels. The matrix of weights also known as the correlation kernel is not rotated while doing the computation. Hence it results in a value of how a pixel is correlated to its neighboring pixel.

Correlation $=\Sigma[(i-\mu i)(j-\mu j)(\sigma i * \sigma j)]_{N-1 i, j=0}$

After getting these texture features, the set of statistical features is computed from the segmented blood vessels, exudates, and microaneurysm images. The statistical features computed are an area, perimeter, eccentricity etc. The feature vector is a combination of both texture and statistical features. The features calculated above are then given as input to the machine learning classifiers for the classification of the input image.

\section{Classification}

Machine learning has many algorithms for classification and regression. Supervised learning problems like classification have two stages of training and testing. In the training stage, the classifier generally learns the set of images which are known. In this paper, the feature vector generated by GLCM and the other features for every single image is provided to Decision tree and other classifiers with their respective labels. In the testing stage, unknown image is given to the classifier for getting the classification results. Decision tree classifier recurrently splits the input dataset or the area into small sub-portions according to a particular parameter. It is able to manage both categorical and numerical variables. This is one of the fastest methods to recognize the most significant parameters and the relation between them. In Logistic regression classifier, the dependent variable is generally categorical. The output value represents various classes. AdaBoost classifier, the name is derived from Adaptive Boosting. This classifier initially predicts the given input data and assigns equal weight values to each observation. It tries to fit a series of weak learners (other learning algorithms) on the various training dataset. AdaBoost can be used for regression and classification problem. Feedforward artificial neural network which is a multilayer perceptron has minimum three layers. It contains an input layer, a hidden layer, and the output layer. The output layer is used to make the predictions over the input data. Again, the network uses backpropagation which is a supervised learning concept.

\section{Results and Discussion}

The dataset contains total 1047 images which have a combination of all normal, NPDR and PDR images. The whole dataset is split into 80-20 ratio while performing training and testing of the classifiers. The testing process has been done on 210 images. The features which are generated by using the above methods are fed to the classifier and got the results. Decision tree classifier gave $74.28 \%$ accuracy, the confusion matrix of this classifier is shown in Figure 7. Again in binary classification, it gave $82.85 \%$ accuracy. Logistic regression and AdaBoost classifier resulted in $54.28 \%$ and $50.95 \%$ respectively. Here 0 represent Normal class and 1 and 2 shows NPDR and PDR classes respectively. The artificial neural network gave $66.79 \%$ accuracy, here we used 3 hidden layers and 'SGD' optimizer which controls the learning rate. The activation function used for the hidden layer is ' $\mathrm{ReLu}$ ' which is Rectified Linear Unit. Finally, the output layer generates the result according to the input weights. Its confusion matrix is shown in Figure 9. Figure 10 shows the performance comparison of all the three classifiers based on the accuracy. There is always a room for improvement, our future implementation includes the application of convolutional neural network (CNN) which is a part of deep learning. CNN does both feature extraction and classification which is used improve the results.

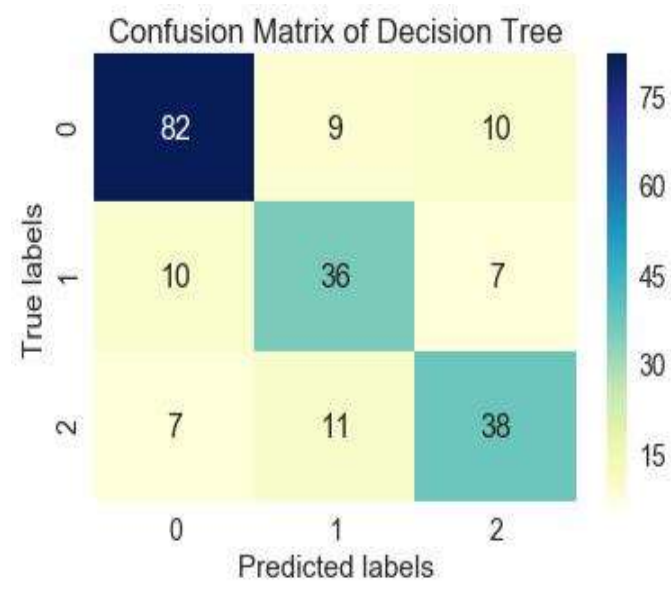

Figure 7. Decision Tree Confusion Matrix for Multiclass Classification. 


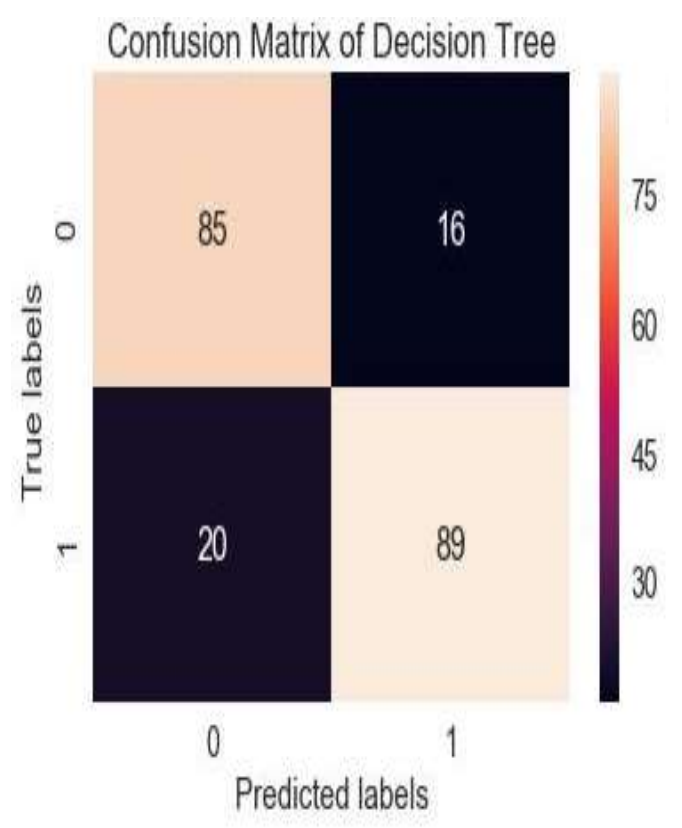

Figure 8. Decision Tree Confusion Matrix for Binary Classification

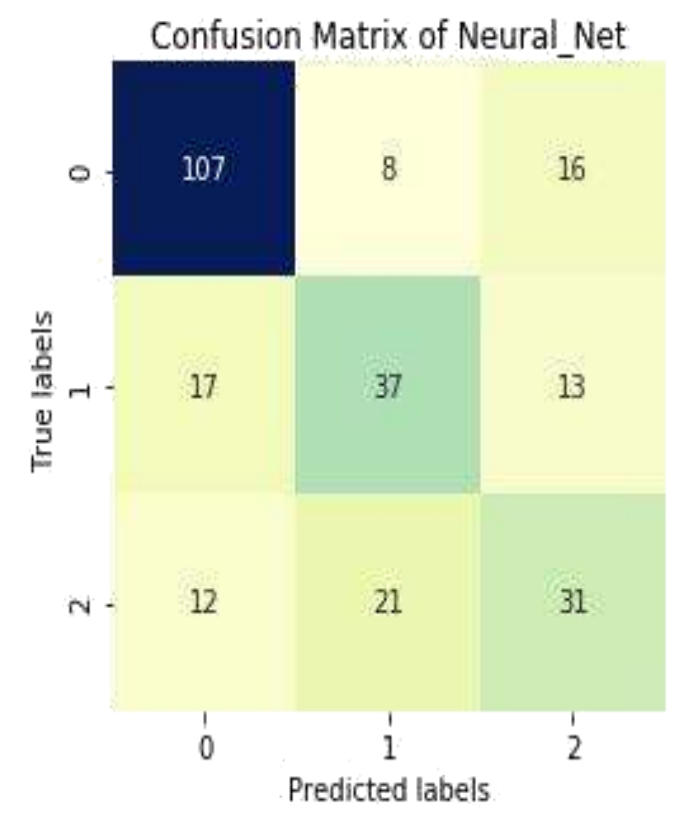

Figure 9. ANN Confusion Matrix for Multiclass Classification.

\section{Performance of Classifiers}

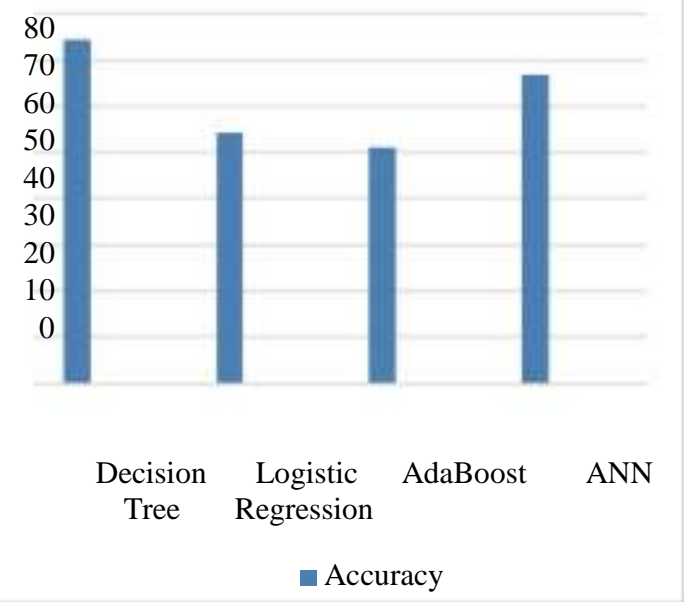

Figure 10. Comparison of all Classifiers

\section{Conclusion}

Diabetic retinopathy is a critical issue. This paper suggested different machine learning classifiers to identify the affected images. First, the input image is pre-processed and various features have been segmented by using image processing operations. Later, the feature vector is generated and classification is done. Three classifiers are used for classifying the input images such as decision tree, logistic regression, and AdaBoost. From the obtained results it is observed that the Decision Tree classifier outperformed the other three models. Decision tree gave $74.28 \%$ accuracy and ANN gave $66.79 \%$. However, the efficiency of classification process can further be improved with some better feature values. The future work includes improving the performance of the proposed system by using the other machine learning classifiers to get more accurate results. We will attempt to increase the results by using some other deep learning techniques to get accurate feature values and classification outcome.

\section{References}

1. G.Gardner, D.Keating, T.H.Willamson, A.T.Elliott (1996), "Automatic detection of diabetic retinopathy using an artificial neural network: a screening Tool", Brithish Journal of Opthalmology ;80:940-944.

2. D. Vallabha, R. Dorairaj, K. R. Namuduri and H. Thompson (2004), "Automated Detection and Classification of Vascular Abnormalities in Diabetic Retinopathy", 38th Asilomar Conference on Signals, Systems and Computers, Vol. 2, pp. $1625-1629$. 
3. S.Roychowdhury, D.D.Koozekanani, Keshab K.Parhi(2014), "DREAM:Diabetic Retinopathy AnalysisUsingMachine Learning", IEEE Journal of BioMe dical and Health Informatics, Vol.18, No 5.

4. Diego Marín, Arturo Aquino, Manuel Emilio Gegúndez-Arias, and José Manuel Bravo(2011). "A New Supervised Method for Blood Vessel Segmentation in Retinal Images by Using Gray-Level and Moment Invariants-Based Features," IEEE TRANSACTIONS ON MEDICAL IMAGING, VOL. 30, NO. 1.

5. Akara Sopharak, Bunyarit Uyyanonvara, Sarah Barman (2009), "Automatic detection of diabetic retinopathy exudates from non-dilated retinal images using mathematical morphology", Computerized Medical Imaging and Graphics, Vol. 32(8), pp 720-727.

6. R.G Ramani, L.Balasubramanian, S.G.JacobROC (2013), "Analysis of classifiers in automatic detection of Diabetic Retinopathy using shape features of fundus images", International Conference on Advances in Computing, Communications and Informatics (ICACCI).

7. Jaydeep De, Li Cheng, Xiaowei Zhang, Feng Lin, Huiqi Li, Kok Haur Ong, Weimiao Yu, Yuanhong Yu, Sohail Ahmed (2016), "A GraphTheoretical Approach for Tracing Filamentary Structures in Neuronal and Retinal Images”. IEEE Transaction on Medical Imaging, Vol. 35, No. 1, pp 257-272.

8. R.Priya, P.Aruna (2013), "Diagnosis of diabetic retinopathy using machine learning techniques", Ictact Journal On Soft Computing, Volume: 03, Issue: 04

9. Mahendran Gandhi, R. Dhanasekaran (2013), "Diagnosis of Diabetic Retinopathy Using Morphological Process and SVM Classifier” IEEE International conference on Communication and Signal Processing, April 3-5, India

10. Michael D. Abràmoff, Mona K. Garvin, Milan Sonka (2010), "Retinal Imaging and Image Analysis", IEEE Reviews in biomedical engineering, vol. 3, pp 169-208.

11. J. Nayak, P. Bhat, U. R. Acharya, C. M. Lim, Kagathi (2008), “Automated identification of diabetic retinopathy stages using digital fundus images,” J. Med. Syst., vol. 32, pp.107-115.

12. M.R.K. Mookiah, U. R. Acharya, R. J. Martis, K. Chua, L. C. Min, E. Y. K. Ng, A.Laude (2013), "Evolutionary algorithm based classifier parameter tuning for automatic diabetic retinopathy grading: a hybrid feature extraction approach," Knowl. Based Syst., vol. 39(0), pp. 9-22.

13. Mookiah, M.R.K., Acharya, U.R., Chua, C.K., Lim, C.M., Ng, E., Laude, A (2013), "Computer-aided diagnosis of diabetic retinopathy: A review". Computers Biology and Medicine, 43(12):2136-2155.
14. M.Rajesh Babu, BVNR Siva Kumar, Rakeshumar (2016), "Diagnosis of Diabetic Retinopathy Using Morphological Process and SVM Classifier", | IJIRT | Volume 3 Issue 4 |ISSN: 2349-6002.

15. Pooja M. Pawar and Avinash J. Agrawal, "Developments for Diabetic Retinopathy Detection and Classification: A Review", International Conference on Recent Trends in Engineering \& Sciences (ICRTES), February 21, 2018. [Presented]. To be published in coming issues Vol 8 of International Journal of Engineering \& Technology (UAE) [SCOPUS Indexed]. 